\title{
Out of theatre sedation - how I do it safely
}

\section{T Hlongwane-Gukuta}

Specialist Anaesthetist, Chris Hani Baragwanath Academic Hospital, University of the Witwatersrand Corresponding author, email: thenjiweh@yahoo.co.uk

\section{Introduction}

Over the last few years I have had the pleasure and privilege of providing procedural sedation and analgesia (PSA) in a number of locations remote to theatre at Chris Hani Baragwanath Academic Hospital (CHBAH). Of note has been providing PSA for transesophageal echocardiography (TEE) at the cardiology department of $\mathrm{CHBAH}$. This has been a steep learning curve as the high-risk patient profile adds to the stress of working in what is already a daunting environment and 'anaesthetic'. Through this experience, I have learnt much about the safe practice of PSA outside of the operating theatre environment.

This article will summarise the lessons learnt using my experiences to emphasise the prerequisites for the safe practice of PSA in remote locations. I will start with a brief discussion of basic concepts and then move on to how we have adhered to local and international guidelines to ensure safety in our environment.

\section{What is Procedural Sedation and Analgesia (PSA)?}

PSA exists along a continuum in terms of consciousness from anxiolysis or minimal sedation to deep sedation and analgesia. ${ }^{1,2}$ Short acting analgesic and sedative drugs are utilised to create conditions conducive to optimal performance of therapeutic or diagnostic procedures. ${ }^{1}$ A detailed discussion of each drug is beyond the scope of this article.

As the level of sedation and analgesia deepens along the continuum, increasing depression of the cardiovascular and respiratory systems results and with it, the risk of adverse events increases. The sedationist must be skilled and have access to all equipment necessary to manage any complications quickly and effectively.

The South African Society of Anaesthesiologists (SASA) published sedation guidelines in 2015. In them, SASA identifies the following levels of sedation as defined by the American Society of Anesthesiologists (ASA) ${ }^{2}$ :

\section{Minimal sedation and anxiolysis}

In this drug-induced state, patients can respond normally to commands. Airway reflexes, respiratory and cardiovascular function is unaffected although mentation and coordination may be impaired.

\section{Moderate sedation and analgesia}

This refers to the classic term 'conscious sedation'. In this drug-induced state, patients respond purposefully to verbal commands with or without light tactile stimulation. Usually, patients maintain their airway and ventilation with no intervention. Haemodynamic stability is usually maintained.

\section{Deep sedation and analgesia}

This is also known as 'monitored anaesthesia care'. In this druginduced state, patients are not easily rousable but may respond purposefully to repeated or painful stimuli. Note that reflex withdrawal to pain is NOT purposeful. Ventilatory depression is usual at this level and the sedationist may be required to assist in airway maintenance and ventilation.

\section{General anaesthesia}

Here, loss of consciousness is induced and patients are not rousable at all. Assistance is required to maintain the airway and with ventilation. Cardiovascular function may require support.

In general at $\mathrm{CHBAH}$, we have occasion to employ minimal to deep sedation in our remote locations. Because we recognise that a patient may end up in a deeper plain of sedation than intended, all our remote locations are equipped with everything required to 'rescue' a patient from a deeper plane of sedation than intended or indeed perform Advanced Cardiac Life Support (ACLS) should a patient require resuscitation.

\section{What is a remote location?}

A remote location is any location other than an operating theatre where sedation or general anaesthesia is administered. In the United States of America, anaesthesia or sedation in remote locations accounts for $12.4 \%$ of anaesthetic care. ${ }^{6}$ 
At $\mathrm{CHBAH}$, we provide PSA at the following remote locations as described in the literature cardiology (TEE, cardioversion, cardiac catheterization lab), audiology (auditory evoked potentials) and radiology (neurointerventional radiology, vascular radiology, $\mathrm{MRI} / \mathrm{CT}, \mathrm{PET}$ scan). ${ }^{2}$ Other locations mentioned in the literature include dental surgery, endoscopy suite (Gastrointestinal suite), fertility surgery, dermatology, plastic surgery and the emergency medicine department. ${ }^{2}$

Sedation should only be performed where facilities, personnel, equipment and drugs needed to manage emergencies are immediately available. Resuscitation equipment must be checked regularly and maintained. ${ }^{2}$ Safe sedation practice should be maintained at all times by ensuring appropriate patient selection and adequate pre-procedure assessment and monitoring is available. ${ }^{2}$ Regardless of the location, the sedation team should have access to an intensive care setting should complications that warrant its use arise.

The SASA PSA guidelines provide a comprehensive of the basic equipment and drugs for procedural sedation and analgesia in adults. ${ }^{2}$ In summary, these include ${ }^{2,6}$ :

- Reliable oxygen source

- Suction apparatus

- Waste gas scavenging

- Adequate monitoring equipment

- Safe electrical outlets

- Adequate lighting and battery backup

- Sufficient space for anaesthesia personnel and equipment

- Emergency trolley, defibrillator and drugs

- Reliable two way communication

- Safety codes met

- Appropriate post anaesthesia management

\section{Why PSA?}

The aim of procedural sedations is twofold: firstly, to reduce discomfort and anxiety associated with a procedure and secondly, to facilitate the procedure to ensure the desired outcome be it therapeutic or diagnostic. ${ }^{1}$ In accomplishing these aims, patient cooperation and physiological stability need to be maintained. ${ }^{2}$ All of the above can be accomplished using pharmacological and non-pharmacological methods. ${ }^{2}$

Provided that patient and procedure selection are done appropriately to reduce risk, there may be a cost saving to performing certain procedures under PSA in a remote locations. ${ }^{3}$ This is because the costs of theatre time and hospital admission can be avoided.

\section{Who should get PSA?}

Along with adequately equipped setting for performing sedation, good patient selection is critical to ensure safely for PSA.

There is no absolute contraindication to PSA. ${ }^{1}$ Relative contraindications include: extremes of age, significant comorbidities (ASA III or more), possible difficult airway, obesity and high risk of aspiration. ${ }^{1}$ In most cases, the sedation poses more risk to the patient than the procedure itself making careful patient selection critical. $^{2}$

Four main considerations inform the decision to select a patient for procedures under PSA:

\section{The prodecure}

PSA can be employed to facilitate therapeutic or diagnostic procedures that do not require neuromuscular blockade or unconsciousness. ${ }^{2}$ The duration, level of discomfort, patient positioning and surgical support influence patient selection. ${ }^{6}$ Sedation experts recommend that any sedation procedure lasting more than four hours done in a remote location should be defined as prolonged sedation. ${ }^{2}$ It is recommended that a procedure expected to be more than four hours in duration be performed under general anaesthesia in the hospital.

\section{The patient}

The patient should be counseled and informed consent obtained. The SASA sedation guidelines provide a comprehensive history questionnaire. ${ }^{2} \mathrm{~A}$ detailed sedation history should also be elicited. A focused examination should be performed with particular emphasis on airway evaluation. Comorbidity, airway assessment, fasting status and monitoring requirements need to be considered.

\section{The operator}

The operator should be comfortable with performing the procedure under PSA.

\section{The environment}

Adequate equipment and staff should be immediately available to assist the patient should complications arise.

If advanced sedation techniques are planned, standard anaesthetic fasting guidelines are recommended namely: no clear fluids for two hours and no solids for six hours prior to the procedure. $^{2}$

The cardiologists at $\mathrm{CHBAH}$ require sedation for their more complex TEEs to facilitate obtaining good quality diagnostic information. The challenge with this patient profile is that in most cases they are ASA III or more by virtue of advance cardiac disease. We mitigate the risk of such sedation by sending anaesthesiologists experienced in providing sedation for patients with cardiac disease, as they are able to offer the support the patient requires should cardiovascular compromise occur.

\section{What types of sedation techniques can be employed ${ }^{2}$ ?}

Simple or advanced sedation can be provided to a patient depending on the skill of the sedationist. ${ }^{2}$ Simple or standard sedation is induced using one drug. ${ }^{2}$ The addition of another drug renders it an advanced sedation technique. Thus, advanced sedation includes any combination of drugs by any route, sedation via the intravenous route, any inhalational sedation (excluding $\mathrm{N}_{2} \mathrm{O}$ in a concentration less than $50 \%$ ) and infusion techniques. $^{2}$

The main factors determining the choice of sedation technique as recommended by the SASA sedation guidelines ${ }^{2}$ are: 
- The risk:benefit ratio of the technique.

- The requirement for prolonged sedation, especially as more procedures are being performed outside the operating theatre.

- The characteristics of the patient, e.g. ASA classification and risk assessment profile.

- The nature of the procedure being performed, e.g. painless or painful, and major or minor.

- The availability of skilled personnel to monitor and perform the procedure.

- The availability of skilled support staff to assist, should rescue be necessary.

- The environment and clinical setting, e.g. premises, drugs and equipment.

- The qualifications, skills and experience of the seditionist.

- Contraindications.

- Whether or not it is evidence based.

At CHBAH hospital, we generally use advanced sedation techniques by virtue of the fact that as anaesthesiologists, we are skilled and equipped to manage deeper planes of sedation that may occur.

\section{What are the adverse consequences of sedation?}

Serious morbidity as a result of PSA is rare. ${ }^{4,5}$ Adverse events are more likely in remote locations and occur for a number of reasons (quoted from the SASA guidelines $2015^{2}$ ) including but not limited to:

- Inadequate patient selection, inadequate preoperative assessment and poor preparation

- The effects of sedatives and analgesics, especially if used in combination

- Timing of the administration of drugs

- Inadequate knowledge of the pharmacokinetics and pharmacodynamics of drugs

- An unanticipated pharmacogenetic response to drugs

- Inadequate monitoring of the patient

- The inability of the sedation practitioner to manage complications

- The inability of the sedation practitioner to rescue a patient from an unexpected or undesirable deeper plane of sedation

- The premature discharge of patients who have not met the discharge criteria after sedation.

Adverse events associated with PSA include ${ }^{6}$ :

- Airway

- Airway obstruction

- Aspiration

- Regurgitation

- Dental/soft tissue injury

- Respiratory

- Respiratory depression

- Hypoxaemia

- Hypercarbia

- Apnoea
- Cardiovascular

- Hypotension

- Arrhythmias

- Neurologic

- Deeper level of sedation than desired

- Unresponsiveness

- Other

- Undesirable patient movement

- Drug interactions

- Adverse reactions

- Unanticipated admission

Failed sedation results in an inadequate level of sedation such that the procedure is abandoned or general anaesthesia is needed. ${ }^{2}$ It can be due to any factors pertaining to the patient, drugs, procedure or operator. ${ }^{2}$ Previous failed sedation necessitates a general anaesthetic in future.

At $\mathrm{CHBAH}$ we have skilled anaesthesiologists providing PSA to manage the above complications appropriately and equipment in some of our remote locations to convert to general anaesthesia should sedation fail.

Table 1: The modified Aldrete scoring system

\begin{tabular}{ll}
\hline Characteristics & Score \\
\hline Level of consciousness & 2 \\
\hline Fully awake & 1 \\
\hline Arousable when called & 0 \\
\hline No response & \\
\hline Oxygen saturation (\%) & 2 \\
\hline >90\% breathing room air & 1 \\
\hline Oxygen required to maintain saturation > 90\% & 0 \\
\hline < $90 \%$, even when breathing oxygen & \\
\hline Circulation and blood pressure & 2 \\
\hline Systolic BP within 20 mmHg of the pre-sedation level & 1 \\
\hline Systolic BP within $20-50$ mmHg of the pre-sedation level & 0 \\
\hline Systolic BP > 50 mmHg of the pre-sedation level & 2 \\
\hline Movement and activity & 1 \\
\hline Able to move all extremities on command & 0 \\
\hline Moves 2 extremities & 2 \\
\hline Doesn't move extremities & 1 \\
\hline Respiration & \\
\hline Able to breathe and cough freely & \\
\hline Dyspnoea, or shallow or limited breathing & \\
\hline Apnoea & \\
\hline BP: blood pressure & \\
\hline
\end{tabular}




\section{Discharge criteria}

The patient must be recovered in an adequately equipped recovery room, with a healthcare professional trained in Basic Life Support (BLS) monitoring them. ${ }^{2}$ Only two patients may be monitored by one healthcare professional in the recovery room. ${ }^{2}$ The seditionist assumes overall responsibility for patients recovering from PSA and may not leave the premises until the discharge criteria are met. ${ }^{2}$ The table below illustrates discharge criteria according to the modified Aldrete scoring system²:

\section{Summary}

Procedural sedation and analgesia in remote locations is growing in popularity and complications remain low provided that the right type of procedure is performed on the right type of patient using the right type of sedation technique in the right location. The SASA Sedation Guidelines 2015 provide an excellent framework for providing safe sedation service. Adherence to these guidelines helps to reduce the risk of PSA in remote locations.

\section{References}

1. Frank RL. Procedural sedation in adults. UpToDate 2016.

2. Roelofse J, Piercy J. Guidelines for the safe use of procedural sedation and analgesia for diagnostic and therapeutic procedures in adults: 2015 . South Afr J Anaesth Analg 2015;21(2)S1-S36.

3. Atkinson P, French J, Nice CA. Procedural sedation and analgesia for adults in the emergency department. BJA 2014; 348.

4. Clinical policy for procedural sedation and analgesia in the emergency department. American College of Emergency Physicians. Ann Emerg Med 1998; 31:663.

5. Bellolio MF, Gilani WI, Barrionuevo P, et al. Incidence of Adverse Events in Adults Undergoing Procedural Sedation in the Emergency Department: A Systematic Review and Meta-Analysis. Acad Emerg Med 2016; 23:119.

6. Butterworth JF, Mackey DC, Wasnick JD. Morgan and Mikhail's Clinical Anesthesiology. $5^{\text {th }}$ Edition. 2013. The McGraw-Hill Companies Inc. 